\title{
DEL JEEP WILLYS A LA LLAVE MAYA: MARCAS LEXICALIZADAS EN EL ESPAÑOL DE COSTA RICA
}

\author{
Víctor Ml. Sánchez Corrales*
}

\begin{abstract}
RESUMEN
En el siguiente artículo, teniendo en cuenta los mecanismos de formación de nuevas palabras, especialmente la creación léxica y el préstamo, se presentan y describen lexicográficamente ejemplos de lexicalizaciones de nombres marca en el español de Costa Rica.

Palabras clave: Nombres marca, lexicalización, préstamo lingüístico, español de Costa Rica.
\end{abstract}

\begin{abstract}
**
Considering the mechanisms involved on new word formation, especially lexical creation and lexical borrowing, the following article presents and describes lexicographically some illustrations regarding lexicalizations of brands in Costa Rican Spanish.

Key Words: brands, lexicalization, lexical borrowing, Spanish from Costa Rica.
\end{abstract}

\section{Introducción}

En Costa Rica hubo necesidad de abrigar a los pobres bananeros, muchos de ellos originarios de la tórrida llanura de Nicaragua; y el único abrigo abundante en el país era la tradicional "cobija colorada", que siempre ha cubierto las camas de los campesinos de la Meseta Central costarricense.

Aquella cobija colorada, tan fuera de lugar en los desfiles callejeros, pronto recordó a nuestra gente el sarape que usaban al hombro, en sus visitas o giras artísticas por nuestro pais, los mariachis mexicanos. Asi se convirtieron los soldados gobiernistas en mariachis. Así nacen las palabras.

Por extensión, el término "mariachi" se aplicó durante un cuarto de siglo a cualquier adversario político del Movimiento de Liberación Nacional. Así se extienden las palabras

(Figueres Ferrer, 1977:17).
En efecto, varios son los procesos de formación de palabras nuevas y su incorporación al léxico de la correspondiente lengua, de conformidad con su naturaleza de vida en sociedad en un espacio y tiempo de los hablantes: sus usuarios-creadores son seres en familia, en comunidad, en un entorno físico y cultural, en un momento histórico, que, mediante la lengua, atesoran esa experiencia de vida en su lenguaje, especialmente en su repertorio de palabras. En sentido contrario, también las palabras desaparecen del inventario léxico de una lengua por razones también varias:

* Instituto de Investigaciones Lingüísticas. Lexicografía (ELEXHICÓS). Universidad de Costa Rica.

** Traducción: Licda. Geannette Soto. Escuela de Lenguas Modernas. Universidad de Costa Rica.

Recepción: 19/02/13 Aceptación:13/03/13. 
el lenguaje como sistema de comunicación, como instrumento para hacer cosas y de la interacción lingüística de los miembros de una comunidad entre ellos y para con el entorno, es connatural a una serie de factores culturales, históricos, económicos, de género, etc. Bienes materiales caen en desuso por inoperantes, ideas fenecen, emergen presiones sociales para evitar términos malsonantes, etc., y con ello, mueren los respectivos signos lingüísticos por vacuidad en cuanto a la designación: la mortandad léxica es también connatural a la lengua, cuyas consecuencias se hacen sentir en el inventario léxico de la correspondiente comunidad de habla.

\section{II. ¿Cómo nacen las palabras?}

En este apartado nos preguntaremos sobre el cómo surge una nueva palabra en una lengua dada, cuáles son sus mecanismos de formación, para incorporarse al respectivo repertorio léxico, pues el porqué tiene como razón la necesidad constante de la adecuación de la lengua de la correspondiente comunidad de habla al construir y deconstruir su experiencia de vida, esto es a los usos, costumbres, bienes y servicios, acontecimientos, cambios sociales, producción y comercio, vivienda, clima, accidentes naturales, sistemas educativo y de salud, etc., en que están insertos los miembros de la correspondiente comunidad: “... deberíamos considerar el resultado de la constante incorporación de nuevos términos, y de los nuevos usos dados a los ya existentes, como un signo reconfortante de la vitalidad y creatividad con que una lengua se va formando según las necesidades de sus hablantes" (Yule, 1998:76).

Los hablantes, ante nuevas realidades, de orden material o productos del espíritu, renuevan su léxico, ya a partir de elementos lingüísticos patrimoniales ya provenientes de otras lenguas o mediante la combinación de ambas fuentes. La lengua en su condición de constructo social fluye, en sus distintos elementos lingüísticos, en consonancia con la experiencia de vida de sus hablantes. En este orden de cosas, los hablantes crean palabras para denominar conceptos nuevos, para dar nombre a nuevas realidades; estas palabras en el momento de su génesis son totalmente nuevas y no se han incorporado en el diccionario de una lengua de cultura. Estamos ante un neologismo, elemento lingüístico connatural a la lengua: "el neologismo da vida a la lengua, sirve para renovar su léxico, actualizar elementos, dar cuenta de nuevas realidades, y en él se manifiestan tensiones que tienen lugar en el interior del léxico" (Alvar Ezquerra, 1998:49).

De los diferentes procesos de formación de nuevas palabras (para cuya categorización me baso en Yule 1998:76-83) -derivación (vinazo), composición (lengualarga), mezcla (motel, de "motor" y "hotel"), apócope (profe), retroformación (caries, cariarse), conversión o cambio de categoría (maduro por plátano maduro), acrónimos (ICE : Instituto Costarricense de Electricidad), además de la combinación de procesos: iceísta (sust. /adj. Persona que asume los principios e intereses del Instituto Costarricense de Electricidad)-, nos vamos a referir, por lo pertinente a nuestro trabajo, a la creación léxica y al préstamo.

\section{Creación léxica}

Mediante el proceso de creación léxica, una fuente emisora da vida a un nuevo vocablo y se constituye en su foco difusor entre los miembros de una comunidad de habla, por ejemplo, en el español de Costa Rica la palabra vina sust. /adj. Persona que fisgonea, creada a partir del programa radial de la cómica costarricense Carmen Granados, de grata memoria. Se trata de un término totalmente nuevo en el momento de su aparición respecto del repertorio léxico de los miembros de una comunidad de habla, la costarricense en el presente caso. Los vocablos de mayor vitalidad creados mediante ese proceso, son los que se originan en nombres de marca inventados para algún producto nuevo, servicio o empresa, cuya identidad como nombre (univerbal o pluriuverbal) que designa un referente único está debidamente 
resguardada por un registro legal; este vocablo, con el tiempo y por extensión del contexto, llega a aplicarse para designar cualquier versión de ese producto, por la familiaridad de su uso. En el español tenemos la palabra teflón: $m$. Material aislante muy resistente al calor y a la corrosión, usado para articulaciones y revestimientos así como para la fabricación de ollas y sartenes; proviene del nombre de una marca inventada para designar ese producto, a partir de te(tra)fl(uor)-ón. Es un préstamo del inglés.

Rosenberg, en su Diccionario de administración y finanzas (1994:256), define marca registrada en los siguientes términos: "marca registrada trademark. DER. Distintivo identificativo de un producto manufacturado o de un servicio en forma de nombre, lema, consigna, etc. Toda marca registrada está protegida por ley, y solamente puede ser usada por su titular. En EUA, los organismos que usen una marca por un período de cinco años, siempre que hubieren presentado la solicitud, tendrán derecho al título de la marca registrada. Sin embargo, este derecho se puede perder si el nombre se vuelve genérico. Nombres genéricos son aquellos que los consumidores utilizan para identificar un tipo de productos, más que para indicar uno concreto (por ejemplo, aspirina, nylon, kleenex)".

La Real Academia Española (2001:1450), en los sublemas pluriverbales de la entrada marca, registra lo siguiente: "marca de fábrica. f. Distintivo o señal que el fabricante pone a los productos de su industria, y cuyo uso le pertenece exclusivamente. II marca registrada. $f$. Marca de fábrica o de comercio que, inscrita en el registro competente, goza de protección legal".

Una marca registrada es, por tanto, un símbolo inscrito legalmente ante la instancia institucional correspondiente por una persona física o jurídica, para identificar un producto, un servicio o una empresa y distinguirlos de sus competidores, reales o eventuales, proporcionándoles identidad comercial y resguardando el derecho de uso exclusivo para la persona física o jurídica que ha realizado el registro. El símbolo puede ser una palabra o una frase (marcas denominativas), una imagen o un diseño (marcas figurativas), y las mixtas, que combinan ambos recursos.

En el caso de Costa Rica, de conformidad con la normativa correspondiente (Artículo 47 de la Constitución Política, Ley de Marcas y otros (sic) Signos Distintivos $N^{\circ} 7978$ de 6 de enero de 20000 y su Reglamento), el certificado legal de registro de marca lo emite el Departamento de Propiedad Industrial, dependencia adscrita al Ministerio de Justicia y Gracia.

No obstante lo anterior, un símbolo simple o complejo, por ejemplo una palabra o frase que han sido registradas como marcas comerciales, puede perder la condición de identidad de un producto o servicio concretos, cuando una comunidad de habla lo emplea para referirse a otros de su mismo tipo: "jeep, vehículo de transporte de personas, que tiene un motor muy potente, de llantas de estrías gruesas, de doble tracción, una de cuyas velocidades es baja pero extra fuerte, y que sirve para circular en caminos escarpados, sin asfalto e irregulares". Es un nombre marca porque proviene de la marca denominativa registrada Jeep, y en particular del Jeep Willys, vehículo automotor de fabricación estadounidense y de gran utilidad en la Costa Rica rural de los años cuarenta y cincuenta. El ejemplar más antiguo que se conserva en Costa Rica es un modelo Militar GPW de 1942. Otros vehículos, de características y usos semejantes, pero de marcas distintas pasaron a ser también jeeps: Land Rover, importado por Almacén Electra en 1957 (comunicación personal del señor Héctor Picado Fonseca, ex agente vendedor de la empresa) y Toyota Land Cruiser, importado por H. T. Purdy Inc. (propiedad del señor Amadeo Quirós) también ese mismo año. En la actualidad, además de los jeeps Willys, Toyota y Land Rover anteriormente mencionados, circulan por nuestras calles jeeps Mitsubishi, Kia, Suzuki, Korando, Ssang Yong, etc.

Aunque brevemente, es oportuno referirnos al concepto de lexicalización como un proceso de cambio lingüístico mediante el cual, en el caso que nos ocupa, una marca denominativa pierde tal estatus, para adquirir la condición de elemento lingüístico integrado 
al repertorio léxico de una variedad de lengua determinada, con adecuación a la estructura morfosintáctica correspondiente. Las marcas denominativas participan del nombre propio en lo relativo a la función de particularizar e identificar un referente único (un bien, un servicio o una empresa), pero difieren en lo concerniente a la finalidad de su creación y al procedimiento para estatuir su identidad y proteger el usufructo para su registradorpropietario. Esa condición de etiqueta denominadora de la marca denominativa, puede perderse cuando los usuarios de una variedad de lengua no establezcan la relación de designación con el referente extralingüístico y le construyan socialmente un contenido lingüístico, al emplearlo ya como un nombre común que, como unidad léxica, designa una clase, esto es un conjunto de unidades que tienen propiedades semánticas y sintácticas comunes.

\section{Préstamo lingüístico}

Las lenguas, el español en el presente caso, han adoptado con el correr del tiempo un gran número de palabras de una lengua donante por razones varias; una de las cuales es llenar vacíos léxicos que presentan las lenguas receptoras. Muchos de los nombres marca, en el caso del español de Costa Rica, tienen procedencia extranjera: marcas registradas en la lengua de una comunidad de habla no española identifican bienes y servicios procedentes de economías en expansión y pujanza: canfín, del producto estadounidense Candle fine, zíper de la marca registrada también estadounidense Zipper.

A manera de ejemplo, se presenta a continuación un listado de nombres marca, en su mayoría originarios de una sociedad extranjera con una economía y desarrollo científico-tecnológico de vanguardia, que se han afincado en el español de Costa Rica sin que estén registrados en los dos diccionarios de lengua, publicados en las dos últimas décadas (Quesada Pacheco 1991, cuarta edición de 2007 y Agüero 1996). Teniendo en cuenta los criterios de novedad y de registro lexicográfico (ReyDebove 1971: 100), tales vocablos se categorizan como neologismos. Estamos ante elementos lingüísticos nuevos, de naturaleza léxica, incorporados al uso lingüístico de los hablantes del español de Costa Rica y que deben figurar en un diccionario de lengua.

La nomenclatura consta de diecisiete entradas y el artículo lexicográfico propiamente presenta la siguiente estructura: el lema, seguido de punto, la marca categorial y la definición propiamente dicha. Inmediatamente después se indica cómo se pronuncia mediante la abreviatura PRON:, cuando no es transparente debido a la escritura en la lengua prestataria.

Un segundo componente, en párrafo aparte y ya no de naturaleza lexicográfica, lo constituye la información enciclopédica del nombre marca que se ha lexicalizado.

ampo. (nombre marca) m. Archivador de pasta dura, de cartón, dotado de un mecanismo para sujetar los documentos en resguardo.

En 1965 la empresa Trejos Hnos. Sucs. S. A. creó la sociedad AMPO Ltda. con el objetivo de producir archivadores manuales para oficinas; a partir de la primera letra de cada una de las cuatro últimas palabras anteriores (archivadores manuales para oficina) se creó el acrónimo $\mathrm{AMPO}$, que, como nombre marca, restringió su aplicabilidad al designar un tipo de archivador por excelencia.

boli. (nombre marca) m. Refresco dulce, líquido o congelado, de sabor artificial a frutas como naranja, fresa, mora, etc. empacado en una bolsita plástica tubular. *ser boli loc. v. coloq juv Ser alguien fanfarrón.

La fábrica de Refrescos Boli, con el objetivo de promocionar su producto, patrocinó un torneo de basquetbol en el año 1962: "Hoy se inicia el triangular final del Torneo Copa Refrescos Boli. El partido correspondiente a la fecha será Tennis-Ángeles, un choque que se espera de los buenos" (La Nación, 16 de noviembre de 1962).

cinta scotch. (nombre marca) f. Banda de presión adhesiva transparente. 
Scotch Tape es el nombre comercial de la banda adhesiva fabricada por 3M, compañía estadounidense; la marca Scotch también fue utilizada por la empresa como prefijo en productos como Scotchgard y Scotch(nombre marca)lite y para sus cintas magnéticas audiovisuales. Es un calco de scocht tape.

cornflex. (nombre marca) $\mathrm{m}$. Cereal tostado en forma de hojuelas, hecho de granos de maíz sometidos a un tratamiento especial. Se consume mucho, solo o con leche, en el desayuno.

El doctor John Harvey Kellogg y su hermano Will Keith registraron el producto agroindustrial "Cereales en hojuelas y su proceso de preparación” el 31 de mayo de 1895; la protección por ley fue otorgada el 14 de abril de 1896 con el nombre de Granose. En 1906 empieza la comercialización del producto, ahora producido por la empresa Kellogg's.

goma loca. (nombre marca) f. Pegamento instantáneo y muy resistente a base de cianoacrilato.

En 1958 Kodak patentó un producto a base de cianoacrilato con la marca Eastman \#910; pronto los cianoacrilatos presentan gran demanda y fueron comercializados por empresas distintas con diferentes nombres, uno de ellos krazy glue, producto de la compañía Fuzhou Huayu, de la República Popular de China. Goma loca es un calco de krazy glue.

ipod. (nombre marca) m. Reproductor multimedia de música y videos, portátil y de tamaño pequeño; incluye otras funciones como juegos, calendario, despertador, notas de voz o de texto.

En el año 2001 la compañía norteamericana Apple Inc. presenta por primera vez el reproductor multimedia portátil iPod. Actualmente se pueden adquirir ipod de diferentes marcas. Es un préstamo léxico (significante y significado) de iPod.

jeep. (nombre marca) $\mathrm{m}$. Vehículo de trabajo y de transporte de personas, que tiene un motor muy potente, con llantas de estrías gruesas, de doble tracción, una de cuyas velocidades es baja y extra fuerte (chancha), y que sirve para circular en caminos escarpados, sin asfalto e irregulares.

Jeep es una marca comercial propiedad del grupo empresrial estadounidense Chrysler LLC, que fabrica vehículos automotores de diferentes tipos; como nombre marca proviene de la marca registrada Jeep, y en particular del Jeep Willys, vehículo de gran utilidad en la Costa Rica rural de los años cuarenta y cincuenta.

kleenex. (nombre marca) m. Pañuelo desechable de papel.

La corporación Kimberly-Clark creó el primer pañuelo facial en 1924, como producto para limpieza del maquillaje, pero a los pocos años su uso se extiende contra el resfriado común y la rinitis alérgica, para que adquiera gran popularidad a partir de los años treinta del siglo XX. Préstamo

kotex. (nombre marca) f. Especie de almohadilla, generalmente de celulosa para absorber el flujo menstrual.

Kotex es una marca comercial de productos higiénicos para la mujer, especialmente durante su período menstrual, propiedad de la compañía estadounidense Kimberly-Clark. Dichos productos devienen muy conocidos a partir de la década de los veinte del siglo pasado, al anunciarlos la compañía en la revista Good Housekeeping.

licra. (nombre marca) f. Prenda de vestir, de material elástico, ceñida al cuerpo, de uso por la mujer, que se ajusta a la cintura, llega generalmente al pie, cubriendo cada pierna por separado. 2. Tela elástica. II*punto licra m. Tejido de algodón y elastano, utilizado en prendas de vestir como pijamas, pantalones, pantalonetas.

En 1959 el químico Joseph Shivers, de la compañía estadounidense DuPont, inventó el elastano o spandex, fibra sintética de gran elasticidad. Ese mismo año DuPont patentizó el producto con la marca LYCRA, cuya propietaria actual es la empresa Invista. El elastano 
revolucionó muchas áreas de la industria textil y hoy en día se utiliza junto con otras fibras para fabricar tejidos de uso vario.

llave maya. (¿nombre marca?) f. Dispositivo computacional de almacenamiento que utiliza memoria flash para guardar información.

La empresa transnacional Audiotrak es fabricante de dispositivos computacionales de grabación y reproducción de audio de alta definición, uno de cuyos productos es MAYA 44 USB, grabadora portátil de audio de gran resolución y de uso profesional. Este tipo de artículos de nombre MAYA, en su condición de USB y forma rectangular, semeja al dispositivo de almacenamiento de memoria, lo que permitió que se ampliara el contexto de uso de la palabra MAYA, ahora para el lápiz de memoria, según el español peninsular. Lo de llave se desopaca por el hecho de que se colgaba del cuello para que no se perdiera, tal como suele ocurrir con personas que así custodian sus llaves. Se trata, en consecuencia, de un cambio semántico del primer constituyente de la lexía compleja y de una lexicalización del nombre marca maya.

maskin (tape). (nombre marca) m. Cinta adhesiva con la parte externa de papel y que se emplea especialmente en trabajos de pintura para proteger lo que no se desea pintar.

En el año 1925, Richard Drew, empleado de 3M, inventó una cinta adhesiva, el masking tape, con el fin de poner al servicio de los trabajadores en pintura de carros un material adhesivo más fácil de retirar una vez realizado el trabajo, sin remover la pintura de las piezas forradas.

metabo. (nombre marca) m. Herramienta de bricolaje o de trabajo, semiautomática, provista de un motor y un engranaje que le permite hacer girar un tornillo (husillo) al que se le acoplan diferentes accesorias en forma de disco y de materiales distintos, según el trabajo que se persiga: lijar, pulir, cortar, cortar metal, madera u hormigón.

Metabo es marca alemana de la empresa METABOWERKE GmbH, fundada en 1924, con asentamiento en Nürtingen, Alemania. Sus primeras producciones de herramientas empiezan dos años después, para constituirse actualmente en una empresa sumamente prestigiosa. Las primeras herramientas de esa naturaleza que se emplearon en el país fueron precisamente de la marca Metabo (comunicación personal de Gustavo Chinchilla y Cristian Godínez, respectivamente maestro de obras y trabajador "media cuchara" en construcción y soldadura).

nintendo. (nombre marca) $\mathrm{m}$. Aparato de juegos y entretenimientos electrónicos que se proyectan en la pantalla de un televisor o en la de una computadora.

La compañía japonesa Nintendo Company Limited es una empresa transnacional de videojuegos, fundada en 1889 y con sede en Tokio, Japón, y constituye la segunda empresa de mayor valor comercial, solo superada por Toyota. Los primeros aparatos electrónicos de ese tipo que se vendieron en el país eran de esa marca.

plastigel. (nombre marca) $\mathrm{m}$. Sustancia pastosa y transparente que se usa para fijar el cabello, dejándolo brillante.

En 1970, Custódio Rangel Pires creó en Brasil, Rio de Janeiro, la empresa Plastigel Industria de Plásticos Rangel Ltda. De ahí el vocablo marca Plastigel, formado a partir del proceso denominado mezcla, identificador comercial de más de 180 productos, pero que en Costa Rica, por reducción de contextos de aplicabilidad, ha adquirido un significado para designar al fijador de cabello, sin reparar en la marca.

tape. (nombre marca) $\mathrm{m}$. Cinta aislante adhesiva que se emplea en los empalmes eléctricos.

En enero de 1946, Snell, Oace y Eastwold, empleados investigadores de la $3 \mathrm{~m}$, patentizaron una cinta de vinil adhesiva como aislante en los acoples eléctricos. Préstamo.

velcro. (nombre marca) $\mathrm{m}$. Sistema de cierre y apertura que costa de dos cintas de tela, una cubierta de fibras enmarañadas y la otra de pequeñas púas flexibles, que se empatan al presionarse. 
Velcro es una marca registrada en el año 1955 por el ingeniero suizo George de Mestral para designar un sistema de cierre y apertura rápido. Es una palabra formada por el proceso morfológico de mezcla: velours (terciopelo) y crochet (gancho), al crearse a partir de las tres primeras letras de esas dos palabras. Actualmente la marca VELCRO está registrada internacionalmente por Velcro Industries B.V., una compañía con sede en Estados Unidos pero con fábricas en distintas partes del mundo.

\section{Comentarios finales}

De los diecisiete espigados del acervo léxico de los costarricenses, doce han sido creados en sociedades de economías pujantes y con el inglés como primera lengua o como lengua internacional de comercio: cinta scotch, cornflex, goma loca, ipod, jeep, kleenex, kotex, licra, llave maya, maskin (tape), nintendo y tape, los cuales representan el 70,58\% del total de los nombres marcas analizados. Las unidades pluriverbales cinta scotch y llave maya tienen, en cada caso, un primer componente aportado por la lengua española; por su parte, si bien los dos componentes de la unidad léxica goma loca también proceden de la lengua española, se trata de un calco de krazy glue. Las otras marcas lexicalizadas: metabo (alemán), velcro (suizo), ampo (costarricense), boli (costarricense y países circunvecinos) y plastigel (brasileña) no se crean desde el inglés y representan el 29,42\% del inventario espigado.

El escaso desarrollo científico y tecnológico del país, la economía de mercado y la globalización, han favorecido la importación de bienes, etiquetados ya en las sociedades de origen y debidamente protegidos los derechos de producción y comercialización. La lengua inglesa es una fuente difusora de marcas registradas en calidad de lengua de comercio internacional. Esas etiquetas, creadas por diferentes procesos, han devenido en nombres comunes al cargarse de contenido semántico.

\section{Bibliografía}

Apple. Apple presents ipod. Disponible e n : ht t p://www.apple.com/pr/ library/2001/10/23Apple-Presents-iPod. html

Audiotrak. Productos. Disponible en: http:// www.audiotrack.net/products/

Albúm de ideas. Disponible en: http:// albumdeideas.blogspot.com/2010/02/ richard-drew-y-3m.html

DRAE en línea. Disponible en: http://lema.rae. es/drae/?val=teflón.

Figueres Ferrer, J. 1977. Así nacen las palabras y los cuentos. San José: Editorial Costa Rica.

El origen del cianoacrilato o "la gotita" o "super glue". Disponible en: http://www. fayerwayer.com/2011/12/el-origen-de-elcianoacrilato-aka-la-gotita-o-super-glue/.

Inventos de la década del cincuenta. Disponible en: http://www.inventos5d.tripod.com/50.html.

Jeep historia. Disponible en: http://www.jeep.es/ extras/historia/1940.html.

Kimbery Clark Perú. Historia. Disponible en: http://kimberly-clark.com.pe/index.php/ historia/en-el-mundo.

LYCRA COM. Acerca de la fibra lycra. Disponible en: http://www.lycra.com/sp_ sp/webpage. $\operatorname{aspx}$ ?id=373.

Metabo. About the metabo. Disponible en: http://www.metabo.de/Die-MarkeMetabo.28+M53dc154aec1.0.html.

Nosotros Ampo, Ltda. Líderes en productos de oficina. Disponible en: http://www. ampos.com/nosotros.php.

Porto Dapena, J. A. 2002. Manual de técnica lexicográfica. Madrid: Arco/Libros, S. A. 
Productos Scoth. Historia de innovación. Disponible en: http://www.scotchbrand.com.ar/wps/ portal/3M/es_AR/GlobalScotchBrand/Scotch/ Resources/Two/.

Rosenberg, J. M. 1994. Diccionario de administración y finanzas. Barcelona: Grupo Editorial Océano.
Wikipedia. Corn flakes de Kellogs. Disponible en: http://es.wikipedia.org/wiki/Corn Flakes_de_Kellogg\%27s

WIRED.com. This day in tech. Disponible en: http://www.wired.com/thisdayintech/2010 /09/0923nintendo-founded/

Yule, G. 1998. El lenguaje. Madrid: Cambridge, University Press. 